\title{
Atomic Force Microscopy of Polymer Systems: From Morphology to Properties to Chemical Imaging and Spectroscopy
}

\author{
Gregory F. Meyers
}

Core R\&D - Analytical Sciences, The Dow Chemical Company, Midland, MI USA

Scanned probe microscopy (SPM) has had a long history at The Dow Chemical Company, beginning in the late 1980s when commercial scanning tunneling microscopes were just hitting the market. Since that time Dow has invested in internal and external collaborative efforts to drive and develop atomic force based technologies for property measurements of polymeric materials at nanometer length scales. The vision of SPM as truly a "lab on a tip" [1] for nanoscale materials research is a key driver for our efforts.

Our most cited early work described a unique phenomenon of surface wear of polystyrene thin films where the interaction of a sliding AFM tip lead to characteristic patterns that depended on the polymer molecular weight [2]. Since that time work such as this has been studied in order to understand elastic, adhesive, and thermal properties of polymer coatings and films near rigid interfaces. Collaborations with NIST in the late 1990s provided our first attempts at quantifying mechanical properties at the nanoscale, combining knowledge gained from classical nanoindentation to the calibration of AFM systems for quantitative AFM based indentation [3,4]. This work set the stage for a large scale effort in the latter 2000 time frame, funded by the NIST-ATP program in collaboration with Veeco Instruments (now Bruker-Nano). The objective of this program was to develop an AFM based platform for quantitative modulus measurements of polymers at sub $100 \mathrm{~nm}$ length scales, to provide better than $10 \%$ precision for polymer materials with bulk properties in the $10 \mathrm{MPa}$ to $10 \mathrm{GPa}$ range. As a result of this program, we now have quasistatic AFM based indentation capability that provides the most comprehensive suite of analytical models (elastic, elastic-plastic, and adhesive) to interpret material properties at these scales [5]. Within the last few years the ability to map properties at high spatial resolution via high speed AFM indenting has been realized [6]. A key consideration in all of the mechanical probing is the time scale over which the tip-surface interaction occurs and so we have looked to computational simulations with Purdue University to study the role of dissipative factors viscoelasticity and surface adhesion hysteresis - to help interpret our measurements.

Concurrent with these developments has been our interest in both measuring and mapping thermal properties of polymer materials using heated AFM probe technology. The transition from micron to nanoscale measurements will be described $[7,8]$.

From 2008-2010 Dow worked with Anasys Instruments on the development of an AFM-infrared (IR) capability (commercialized as the NanoIR in 2010). More recently a top-down version of the system was commercialized (NanoIR2 in 2013). The AFM-IR method relies on detection of IR absorption under the AFM tip by rapid photothermal expansion $[9,10]$. Such an approach breaks the diffraction limit enabling IR mapping at $<50 \mathrm{~nm}$ spatial resolution exceeding that of confocal Raman by $10 \mathrm{X}$ and FTIR by 100X [11]. The method now allows us to 'see' where the chemistry goes in the morphology (Figure 1). 
References:

[1] E. Meyer, H Josef Hug, R. Bennewitz in "Scanning Probe Microscopy: The Lab on a Tip"; $1^{\text {st }}$ Edition (Springer-Verlag, Berlin Heidelberg) 2004.

[2] G. Meyers, B. DeKoven and J. Seitz, Langmuir 8(9) (1992), 2330-5.

[3] G. Meyers, B. DeKoven, M. Dineen, A. Strandjord, P. O'Connor, T. Hu, Y.-H. Chiao, H. Pollock and A. Hammiche, ACS Symposium Series 741(Microstructure and Microtribology of Polymer Surfaces) (2000), 190-211.

[4] M. VanLandingham, J. Villarrubia, W. Guthrie, and G. Meyers, Macromolecular Symposia 167(Recent Advances in Scanning Probe Microscopy of Polymers) (2001), 15-43.

[5] S. Belikov, N. Erina, L. Huang, C. Su, C. Prater, S. Magonov, V. Ginzburg, B. McIntyre, H. Lakrout and G. Meyers, Journal of Vacuum Science \& Technology B 27(2) (2009), 984-992.

[6] C. Su, Microscopy and Microanalysis 16(Suppl. 2) (2010), 364-365.

[7] G. Meyers, M. Dineen, E. Shaffer, T. Stokich, and J.-H. Im, Macromolecular Symposia 167(Recent Advances in Scanning Probe Microscopy of Polymers), (2001), 213-226.

[8] G. Meyers, A. Pastzor, Jr. and K. Kjoller, American Laboratory (Shelton, CT, USA) 39(20) (2007), 9-14.

[9] A. Dazzi, R. Prazeres, F. Glotin and J. Ortega, Optics Letters 30(18) (2005), 2388-2390.

[10] G. Meyers, C. Reinhardt, J. Felts, W. King, C. Prater, D. Cook, and K. Kjoller, 68th Annual Technical Conference - Society of Plastics Engineers (2010), 801-805.

[11] A. Dazzi, C. Prater, Q. Hu, B. Chase, J. Rabolt and C. Marcott, Applied Spectroscopy 66(12) (2012), 1365-1384.
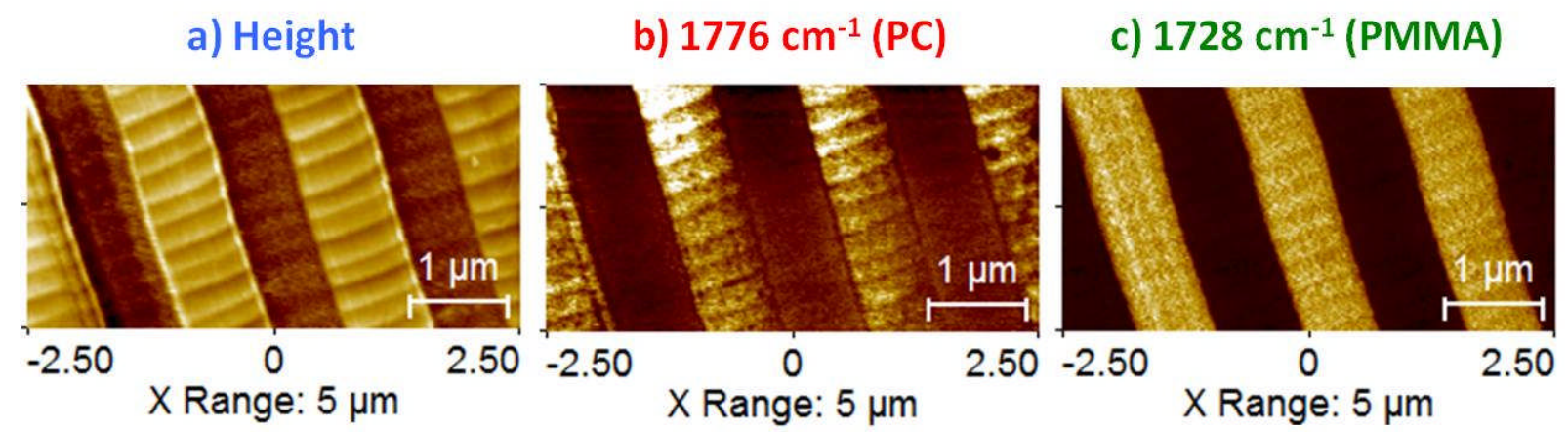

Figure 1. PC $\backslash P M M A$ co-extruded multilayer cross-section showing a) AFM topography; b) AFM-IR map of carbonate functionality; and c) AFM-IR map of acrylate functionality. 